\title{
Estudio comparativo de género sobre el acoso escolar: estrategias y acciones Gender comparative study on bullying: strategies and actions
}

*Alba Bonet-Morro, **Mario Alguacil, **Paloma Escamilla-Fajardo, *Carlos Pérez-Campos, **SergioAguado "Universidad católica de Valencia (España), ${ }^{* *}$ Universidad de Valencia (España)

Resumen. El acoso escolar sigue siendo uno de los problemas más importantes que acontece en el ámbito educativo de países desarrollados. Todavía es un desafío conocer detalladamente los aspectos más importantes para su prevención y tratamiento. Para contribuir a este objetivo, el presente estudio pretende aportar información sobre el acoso escolar y su actuación frente a estas situaciones entre el alumnado, tanto sobre su propia percepción de las situaciones de acoso, como de su actitud frente a dichas acciones sobre otros compañeros. En este sentido, se ha realizado una comparativa para conocer si existen diferencias en estas actuaciones en función el género. Para ello 592 alumnos y alumnas españoles han sido analizados utilizando un instrumento validados previamente por la literatura. Según los resultados obtenidos, existen diferencias significativas entre chicos y chicas en algunas de las cuestiones analizadas sobre el acoso en clase, lo que nos permite conocer más el fenómeno del acoso. Finalmente se han expuesto implicaciones prácticas y estrategias para su posible intervención y tratamiento desde el ámbito escolar.

Palabras clave: Educación, acoso, estrategias educativas, género.

Abstract. Bullying continues to be one of the most important problems occurring in the educational environment of developed countries. It is still a challenge to know in detail the most important aspects for its prevention and treatment. To contribute to this objective, the present study aims to provide information on bullying and its action in these situations among students, both on their own perception of bullying situations, as well as their attitude towards such actions on other classmates. In this sense, a comparative study was carried out to find out if there are differences in these actions according to gender. For this purpose, 592 Spanish male and female students were analysed using an instrument previously validated by the literature. According to the results obtained, there are significant differences between boys and girls in some of the questions analysed on bullying in class, which allows us to know more about the phenomenon of bullying. Finally, practical implications and strategies for possible intervention and treatment in the school environment have been presented.

Key words: Education, Bullying, educative strategies, gender.

\section{Introducción}

Desde hace años, la sociedad se enfrenta a diferentes problemas entre los que destaca el acoso entre iguales (bullying) (Toscano et al., 2020). Este acoso puede tener lugar en cualquier ámbito de actuación, sin embargo, en el ámbito escolar es sin duda uno de los problemas más graves que acontece entre los estudiantes (Bascón-Seda \& Ramírez-Macías, 2020; CastañedaVázquez et al., 2020; Martínez-Baena \& Faus-Boscá, 2018). Esta problemática ha hecho que desde la literatura científica se trate el tema del acoso, intentando dar respuesta por medio de aportaciones cada vez más numerosas que pretenden dar luz a posibles estrategias que permitan atajarlo. En este sentido, podemos encontrar revisiones sistemáticas como la de Martínez-Baena

Fecha recepción: 01-03-21. Fecha de aceptación: 06-10-21

Mario Alguacil Jiménez

mario.alguacil@uv.es y Faus-Boscá (2018) que evidencian ese creciente interés por la temática. En dicha aportación podemos observar como el número de publicaciones relacionadas con el acoso escolar se han multiplicado aproximadamente por 10 en los últimos 20 años. El acoso escolar tiene lugar cuando un alumno o alumna recibe insultos, es ignorado o excluido del grupo, incluso llega a recibir agresiones físicas por parte de sus compañeros (Castañeda-Vázquez et al., 2020). Este puede manifestarse de diferentes formas y es difícil que la víctima pueda defenderse. De ahí que académicos y profesionales hayan dedicado tiempo y esfuerzo en intentar comprender sus causas, consecuencias y acciones o estrategias eficaces para su prevención y tratamiento (Brandão Neto et al., 2020; Fialho \& Bakshi, 2016).

La escuela puede ser el espacio físico y figurado en el que tiene lugar el acoso, sin embargo, la educación también es uno de los medios a través del cual se puede prevenir y actuar para erradicar este tipo de situaciones (Fialho \& Bakshi, 2016). La actuación de la escuela 
como institución y de los profesores como profesionales que tienen un contacto más cercano con el alumnado es vital en la prevención del acoso escolar (Bailey, 2006). Abordar esto desde la Educación Física es fundamental ya que es una asignatura en la que destaca la práctica deportiva y la exposición de habilidades deportivas. En este contexto, según datos macroscópicos, una percepción de competencia física reducida se puede asociar con una mayor probabilidad de sufrir acoso por parte de los compañeros (Bejerot et al., 2013). Por ello, uno de las responsabilidades que tiene el profesorado de Educación Física es generar un clima orientado a la tarea en el que no prime el ego, ya que algunos estudios reflejan la importancia de crear climas de implicación a la tarea y centrados en el alumnado como mecanismo para la prevención de la violencia escolar y la discriminación de género (Menéndez-Santurio, \& Fernández-Río, 2018). En la asignatura de Educación Física la comunicación profesorado-alumnado es más cercana que en otros ámbitos, y quizás esa cercanía nos permite marcar estrategias que ayuden a identificar y actuar ante el acoso escolar dentro de clase, y también contribuir a la prevención de las actuaciones externas relacionadas con el acoso.

A pesar de que existen amplios estudios sobre intervenciones educativas para este fin, no es tan extensa la literatura que aporta información sobre los predictores de éxito de dichas estrategias (Wachs et al., 2019). Para elaborar estas propuestas de intervención en el ámbito educativo es importante conocer la situación del aula, y una de las características principales es conocer si existen diferencias en el acoso escolar y su actuación por parte de los compañeros dependiendo de características individuales. Existe literatura previa que analiza las diferencias en la vulnerabilidad de sufrir acoso escolar y el acoso recibido según el género (Larrain \& Garaigordobil, 2020). Sin embargo, consideramos que también es interesante conocer si existe diferencias en la actuación frente a estos comportamientos de acoso entre chicos y chicas adolescentes. Esto puede aportar información relevante para crear propuestas de intervención en base a esta ello.

Los objetivos principales de nuestro estudio son (i) conocer si existen diferencias significativas en el acoso escolar y la actuación por parte del alumnado ante el acoso según el género, y (ii) exponer implicaciones prácticas y propuestas pedagógicas para abordar la prevención y actuar contra el acoso escolar en Educación Secundaria. La estructura que sigue el artículo tras la presente introducción comienza con un breve marco teóri- co, seguido del método, los resultados obtenidos, la discusión en la que se incluyen implicaciones prácticas y unas conclusiones finales extraídas.

\section{Marco teórico}

\section{Acoso escolar: antecedentes y conceptualización}

El acoso y la violencia no solo es un aspecto que ocurre en el ámbito familiar, sino que también tiene lugar en otros ámbitos sociales, entre los que se encuentran los centros educativos (Navarrete-Galiano, 2009) o el ámbito deportivo (Marracho et al., 2021). Estos mismos autores evidenciaron que los deportistas perciben una necesidad de análisis del acoso, para establecer programas de prevención e intervención en el contexto deportivo y escolar. acoso debe ser un tema crucial en las investigaciones científicas, así como en los programas de prevención e intervención en el contexto deportivo y escolar.

Numerosos hechos ocurridos a lo largo de estos años hasta la actualidad, y su repercusión mediática, han puesto en relieve las graves consecuencias que puede acarrear el hecho de que un adolescente sufra acoso escolar (Wachs et al., 2019). De hecho, estos graves hechos han sido un elemento que ha ido sensibilizando a la sociedad, de forma que ciertas acciones que anteriormente eran pasadas por alto, posteriormente han sido atendidas, debido a esa concienciación sobre las consecuencias que podían acarrear para los adolescentes acosados (Gómez, 2009). Según Ballesteros (2018), en España se atienden alrededor de 25.000 llamadas al teléfono de atención al acoso escolar, de las cuales cerca de 9.000 atienden de forma concreta a situaciones de acoso escolar.

El acoso escolar puede estar hostigado por parte de una persona individual o por un grupo de personas que actúan con el mismo fin. De ahí, hay que destacar que el acoso escolar ocurre en el momento en el que un estudiante sufre de manera continua y durante un amplio periodo de tiempo acciones negativas propiciadas por uno o varios estudiantes. Ocurre cuando el acto es intencional, repetitivo y con una alteración del poder (Olweus, 1998). Por consiguiente, en concordancia con Olweus, acoso escolar o bullying puede ser definido como «la conducta agresiva, intencional y mantenida en el tiempo, perpetrada por un individuo o grupo de ellos contra otro, al que consideran su víctima habitual» (Cerezo-Ramírez, 2012, p. 24). Generalmente se considera que el acaso escolar se produce tras malas conductas repetidas (Shiakou et al., 2019).

La escuela debería de ser un lugar en el que todos 
los estudiantes se sintiesen seguros, sin embargo, en ocasiones se convierte en un lugar amenazador en el cual existe riesgo de sufrir agresiones físicas, emocionales o psicosociales. La reacción de las personas ante este tipo de situaciones es fundamental, ya que justificar o normalizar el acoso como acciones comunes de niños puede ser un grave problema (Knauf et al., 2018; Voors, 2005). Pero además, no solo debemos centrarnos en la reacción, sino que se debe tener claro que la prevención adecuada es la vía que permitirá ayudar para evitar los problemas que pudieran surgir (Vega-Osés y Peñalva-Vélez, 2018).

\section{Actuación ante el acoso escolar}

El papel de los adultos es imprescindible para evitar el acoso escolar (Jiménez-Barbero et al., 2020). El profesorado de los centros educativos tiene la responsabilidad de intervenir ante estas situaciones. Su prevención y tratamiento ha sido foco de atención para académicos y profesionales en los últimos años, de ahí que se haya tratado de determinar unas pautas u orientaciones para disminuir la violencia, diseñando, aplicando y evaluando programas de intervención (Gaffney et al., 2019; Limber et al., 2018). Existen numerosos programas que han sido creados para luchar por la prevención del acoso escolar como «NoTrap!», «KiVa Anti-bullying Program» o «Olweus Bullying Prevention Program»y han sido desarrollados en países y contextos diferentes (Garandeau et al., 2021; Green et al., 2020; Zambuto et al., 2020). Sin embargo, en repetidas ocasiones los padres y profesores desconocen estas situaciones de acoso, ya que la víctima siente vergüenza de ello y no lo comunica, quizás por el miedo que sienten a las represalias que puedan tomar contra ellos las personas agresoras (Alonso et al., 2017).

De ahí que sea muy importante concienciar al alumnado de la actuación responsable que debe de tener ante este tipo de situaciones para favorecer una prevención eficaz y una actuación efectiva. Para ello, como docentes debemos intentar que haya una comunicación fluida con los alumnos para guiarlos de manera que resuelvan los conflictos que les puedan surgir (Izquierdo, 2017). Una forma de prevenir este acoso escolar es tratar en las aulas temas como las normas de convivencia, el desarrollo interpersonal y la colaboración entre compañeros. Según Fernández \& Vivar (2017), la forma de actuar que tendría que tener un profesor debería ser, en primer lugar, llevar a cabo una sensibilización sobre el acoso escolar, para posteriormente realizar un diagnóstico y una prevención. Finalmente, si fuera necesa- ria, una intervención. Tras esto, ha de reflexionar sobre los factores y causas que pueden haber desencadenado la situación y las consecuencias que puede llegar a tener, para intervenir si se cree que la situación lo requiere. No obstante, crear un clima comunicativo y colaborativo donde prime la orientación a la tarea puede fomentar conductas de apoyo ante situaciones de acoso escolar.

\section{Método}

\section{Participantes}

La población de estudio son los estudiantes españoles de educación secundaria y la muestra del estudio está compuesta por un total de 592 alumnos. Del total de la muestra, el $45.3 \%$ son hombres $(n=274)$ y el $52.6 \%$ restante mujeres $(\mathrm{n}=318)$. Todos los alumnos han participado de manera libre en el estudio llevado a cabo, entregando un consentimiento informado y conociendo que el cuestionario que se les ha proporcionado era de carácter totalmente anónimo y con fines exclusivamente académicos. Como criterio de inclusión, se ha establecido que los alumnos hubieran entregado el consentimiento informado y fueran estudiantes de alguno de los cuatro cursos que forman esta etapa educativa, quedando excluidos aquellos que no cumplieran dichos requisitos.

\section{Instrumento}

Como instrumento, se administró el cuestionario sobre abuso entre compañeros elaborado por Fernández y Ortega (1999) que evalúa las situaciones de victimización y el abuso entre el alumnado en el ámbito escolar. El cuestionario consta de 25 preguntas, organizadas en 2 bloques, en el primero se obtiene información acerca de la vida relacional del alumno y en el segundo sobre el abuso entre compañeros que el alumno ha sufrido, infringido o presenciado y su actuación ante ellas. En esta ocasión, basados en el criterio de varios expertos, se han seleccionado algunas de las preguntas consideradas como fundamentales para poder extraer información relevante respecto al abuso escolar.

\section{Procedimiento}

En primer lugar, para poder llevar a cabo el estudio, se contactó con los centros educativos y se solicitó autorización por parte de ellos para poder llevar a cabo la administración de los cuestionarios a los alumnos. Una vez se tuvo esta aceptación por parte de los centros, se 
solicitó un consentimiento informado a los padres/tutores de los alumnos, para posteriormente realizar la recogida de cuestionarios.

El estudio realizado tiene como fin analizar y comparar determinadas características del acoso escolar en centros educativos. La muestra estudiada se seleccionó aleatoriamente dentro de cada centro y el cuestionario fue administrado en horario escolar, de manera anónima y en situación colectiva, habiendo explicando previamente en qué consistía el cuestionario y especialmente su carácter anónimo, por la temática delicada que se analiza. Todos los cuestionarios fueron realizados en papel y posteriormente los resultados se volcaron en el programa estadístico SPSS.

\section{Análisis estadístico}

En primer lugar, se obtuvieron los datos de frecuencias y porcentajes para poder conocer las características sociodemográficas de la muestra. Posteriormente, se empleó el método de chi cuadrado sobre las distintas preguntas del cuestionario en función del género, para conocer la existencia o no de una relación significativa entre las respuestas obtenidas y el hecho de pertenecer a un género u otro.

\section{Resultados}

A continuación, se muestran los resultados del estudio. En primer lugar, respecto a la pregunta «iTe sientes aislado o rechazado por tus compañeros/as desde que empezó el curso?» (ver tabla 1) son las chicas con un $21.7 \%$ las que muestran unos datos superiores en la respuesta «alguna vez» frente al $14.2 \%$ de los chicos. En cambio, si nos fijamos en las respuestas de más de 4 veces o casi todos los días, son los chicos los que tienen un porcentaje levemente mayor.

Una vez realizada la prueba de chi cuadrado, esta ha resultado no significativa $(\mathrm{p}>.05)$ por lo que no podemos decir que exista una relación significativa entre la frecuencia con la que los estudiantes se sienten rechazados y el género al que pertenecen.

En segundo lugar, respecto a la pregunta «¿Es un chico o chica quien se mete contigo?» (ver tabla 2), vemos que las chicas destacan en haber respondido que quién más se meten con ellas son una chica o varias chicas y los chicos por el contrario han respondido que es un chico o varios chicos. Tras el análisis de la prueba chi-cuadrado, confirmamos que existe una relación significativa entre las respuestas y el género $(\mathrm{p}<.05)$ por lo que los resultados sugieren que los chicos reciben más acciones de acoso por parte de los chicos y las chicas por parte de las chicas.

En cuanto a la siguiente pregunta «¿en qué lugares del colegio se meten contigo?» (ver tabla 3), observamos que en las chicas la respuesta mayoritaria es que no se meten con ellas (86.5\%) seguida por en clase (6.9\%) y el patio (3.5\%). En el caso de los hombres, de nuevo la más frecuente es que no se meten con ellos (87.6\%) y de los que sí reciben acoso, estos indican que sucede en cualquier sitio (5.1\%), seguido de en clase (3.6\%) y en el patio (2.9\%). En esta ocasión la prueba chi-cuadrado no resulta significativa $(\mathrm{p}>.05)$ por lo que no podemos confirmar la relación significativa entre el lugar donde se produce el acoso y el género de los estudiantes.

\begin{tabular}{|c|c|c|c|}
\hline $\begin{array}{l}\text { ¿Te sientes aislado o rechazado por tus compañeros/as desde que } \\
\text { empezó el curso? }\end{array}$ & Hombre & Mujer & $\chi^{2}$ \\
\hline Nunca & 82.5 & 76.1 & - \\
\hline Alguna vez & 14.2 & 21.7 & - \\
\hline Más de 4 veces & 2.6 & 1.9 & - \\
\hline Casi todos los días & 0.7 & 0.3 & - \\
\hline \multicolumn{4}{|l|}{$\begin{array}{l}\text { Tabla } 2 . \\
\text { Es un chico o chica guien se mete contigo? }\end{array}$} \\
\hline Es un chico o chica quien se mete contigo? & Hombre & Mujer & $\chi^{2}$ \\
\hline No se han metido conmigo & 86.9 & & - \\
\hline Un chico o varios chicos & 10.6 & 4.7 & * \\
\hline Una chica o varias chicas & & \multirow{2}{*}{6} & * \\
\hline Chicos y chicas & 1.5 & & \\
\hline Todo el mundo & 0.7 & $\begin{array}{c}3.0 \\
0\end{array}$ & - \\
\hline \multicolumn{4}{|l|}{$\begin{array}{l}\text { Tabla } 3 . \\
\text { ¿En qué lugares del colegio se meten contigo? }\end{array}$} \\
\hline ¿En qué lugares del colegio se meten contigo? & Hombre & Mujer & $\chi^{2}$ \\
\hline No se meten conmigo & 87.6 & 86.5 & - \\
\hline En el patio & 2.9 & 3.5 & \\
\hline En el comedor & 0.7 & 0.9 & - \\
\hline En clase & 3.6 & 6.9 & - \\
\hline En cualquier sitio & 5.1 & 2.2 & \\
\hline \multicolumn{4}{|l|}{$\begin{array}{l}\text { Tabla } 4 . \\
\text { ¿Hablas de estos problemas con alguien y le cuentas lo que te pasa? }\end{array}$} \\
\hline ¿Hablas de estos problemas con alguien y le cuentas lo que te pasa? & Hombre & Mujer & $\chi^{2}$ \\
\hline No se meten conmigo & 87.6 & 84.6 & - \\
\hline Con uno o varios amigos & 5.8 & 7.5 & - \\
\hline Con mi familia & 4 & 3.8 & - \\
\hline Con el profesorado & 0 & 1.6 & - \\
\hline No hablo con nadie & 2.6 & 2.5 & - \\
\hline
\end{tabular}

Tabla 5.

¿Te metes $\mathrm{y} / \mathrm{o}$ tratas mal a algún compañero/a?

\begin{tabular}{cccc} 
¿Te metes y/o tratas mal a algún compañero/a? & & & \\
\hline ¿Te metes y/o tratas mal a algún compañero/a? & Hombre & Mujer & $\chi^{2}$ \\
\hline Nunca me meto con nadie & 79.1 & 84.9 & - \\
Alguna vez & 18.7 & 14.8 & - \\
Más de 4 veces desde el comienzo del curso & 1.8 & 0.3 & - \\
Casi todos los días & 0.4 & 0 & - \\
\hline
\end{tabular}

Tabla 6.

¿Si tú intimidas o maltratas a algún compañero/a, por qué lo haces?

\begin{tabular}{cccc}
\hline ¿Si tú intimidas o maltratas a algún compañero/a, por qué lo haces? & Hombre & Mujer & $\chi^{2}$ \\
\hline Nunca me meto con nadie & 77.7 & 88.4 & ${ }^{*}$
\end{tabular} Por gastarle una Por gastarle una brom Porque a mi me lo bacte Porque me ha provocado Porque es más débil o distinto

$\begin{array}{cc}11.3 & 5.7 \\ 1.5 & 1.6\end{array}$

$1.5 \quad 1.6$
$\begin{array}{ll}8.4 & 4.4 \\ 1.1 & 0.0\end{array}$

Tabla 7.

Llegada la ocasión ¿̨crees que tú también podrías tratar mal o amenazar a algún compañero/a? ¿Crees que tú también podrías tratar mal o amenazar a algún $\quad$ Hombre Mujer $\chi^{2}$ compañero/a?
Nunca lo hago ni lo haría Lo haría si estoy con un grupo que lo hace Posiblemente, si me incordia Creo que sí lo haría Seguro que lo haría

$\begin{array}{ll}77.7 & 88.4 \\ 11.3 & 5.7\end{array}$
$\begin{array}{ll}71.3 & 88.4 \\ 11.5 & 5.7\end{array}$ $1.5 \quad 1.6$ $8.4 \quad 4.4$ $\begin{array}{ll}1.1 & 0.0\end{array}$

Tabla 8

\begin{tabular}{cccc} 
¿Qué haces tú cuando se meten mucho con un compañero/a? & & & \\
\hline ¿Qué haces tú cuando se meten mucho con un compañero/a? & Hombre & Mujer & $\chi^{2}$ \\
\hline Me meto para cortar la situación & 54.4 & 44.3 & $*$ \\
Informo a alguna persona (familiar, profesores...) & 20.4 & 33.2 & $*$ \\
No hago nada, aunque creo que debería hacerlo & 12.8 & 18.7 & - \\
No hago nada, no es mi problema & 10.9 & 3.5 & $*$ \\
Me meto yo también con la víctima & 1.5 & 0.3 & - \\
\hline
\end{tabular}


Yendo un paso más allá, respecto a con quién hablan los estudiantes sobre estos problemas, vemos en la tabla 4 que tanto los chicos como las chicas tienden a hablarlo con los amigos (5.8\% y 7.5\%, respectivamente) seguidos por la familia (4\% y $3.8 \%$, respectivamente). Resulta llamativo que ninguno de los chicos ha respondido que hable de estos problemas con los profesores, mientras que un $1.6 \%$ de las chicas sí declara hacerlo. A pesar de esto porcentajes diferentes, la prueba de chi cuadrado resulta no significativa $(\mathrm{p}>.05)$, por lo que no podemos confirmar que exista una relación significativa entre el género y las personas con las que deciden hablar del acoso que sufren.

Una vez analizadas las preguntas referidas a recibir acoso, a continuación, pasamos a analizar aquellas que hacen referencia a infringir el acoso a los compañeros. En primer lugar, respecto a la pregunta «iTe metes y/ o tratas mal a algún compañero/a?» (ver tabla 5), vemos que en ambos géneros se alcanza un porcentaje elevado de estudiantes que reconocen no realizar nunca este tipo de acciones. El porcentaje supera el 20\% en los chicos y el $15 \%$ en las chicas, incluyendo a los que alguna vez han realizado este tipo de acciones y los que las han realizado más de 4 veces a lo largo del curso. Sin embargo, no se puede confirmar que exista una relación significativa entre el género y la frecuencia con que infringen el acoso ( $\mathrm{p}>$.05).

Posteriormente, respecto a la pregunta «Si tú intimidas o maltratas a algún compañero/a epor qué lo haces?», como se puede ver en la tabla 6 , en el caso de los hombres y de las mujeres, los principales motivos son los relacionados con hacer esas acciones como broma (11.3\% y $5.7 \%$, respectivamente) y como respuesta a una provocación $(8.4 \%$ y 4.4 , respectivamente). También se observa que la proporción de mujeres que declara no meterse nunca con nadie es mayor que en el caso de los hombres. En este caso la prueba de chi cuadrado ha resultado significativa $(\mathrm{p}<.05)$, por lo que sí se puede decir que existe una relación significativa entre el género de los estudiantes y el nivel con el que se meten con los compañeros, así como los motivos por los que lo hacen.

La siguiente cuestión que se planteaba a los alumnos era «llegada la ocasión ¿crees que tú también podrías tratar mal o amenazar a algún compañero/a?» (ver tabla 7). Alrededor del $80 \%$ de los hombres y el $84 \%$ de las mujeres declara que no lo haría, sin embargo, también existe un $15.4 \%$ de hombres y un $13.9 \%$ de mujeres que dice que podría hacerlo si le incordian y un $11.3 \%$ de hombres y un $5.7 \%$ de mujeres que afirman que lo harían si es con un grupo que lo hace. Finalmente, un $2.6 \%$ de los hombres y un $1.3 \%$ de las mujeres declara que cree que sí lo haría, siendo prácticamente inexistente el porcentaje de los que aseguran que lo harían. La prueba de chi-cuadrado permite confirmar una relación no significativa entre la variable de género $\mathrm{y}$ las acciones mencionadas $(\mathrm{p}>.05)$

Para terminar con el análisis comparativo en función del género, se ha analizado la pregunta «¿Qué haces tú cuando se meten mucho con un compañero/a?» cuyos resultados se muestran en la tabla 8 . Los hombres muestran un mayor porcentaje en los casos que hacen referencia a meterse para cortar la situación (54.4\%) respecto a las mujeres (44.3\%). Lo mismo ocurre con el enunciado de que no hacen nada porque no es su problema, con un porcentaje mayor en los hombres (10.9\% y 3.5\%, respectivamente). Por otro lado, las mujeres obtienen un mayor porcentaje en cuanto a informar a familiares y/o profesores (33.2\% frente a 20.4\%) y en cuanto a declarar que no hacen nada, aunque creen que deberían hacerlo (18.7\% frente a $12.8 \%$ ). Estos porcentajes diferentes han sido confirmados con la prueba de chi-cuadrado $(\mathrm{p}<.05)$, por lo que podemos confirmar que el hecho de ser hombre o mujer está relacionado significativamente con reaccionar de una forma $u$ otra frente a estas situaciones de acoso.

\section{Discusión}

El presente estudio arroja luz sobre si existen diferencias de género en el hecho de sufrir acoso o acosar, de parte de quién procede el acoso, si comunican el problema, los motivos que llevan a hacerlo y la actuación realizan ante una situación de acoso escolar. Según los resultados obtenidos, las chicas reciben acoso escolar de otra chica o varias chicas de forma más significativa, mientras que, por el contrario, los chicos son el foco de atención de otros chicos desde la perspectiva del acoso. Esto ha sido analizado previamente en estudios españoles obteniendo resultados similares (Vélez \& Fernández, 2018). Por ello, desde la educación en valores podría ser interesante fomentar la coeducación entre iguales, ya no sólo priorizando el trabajo entre ambos géneros, sino también desarrollar una concienciación de que entre el mismo género debe de existir igualdad y respeto.

Los motivos de la intimidación o maltrato a compañeros o compañeras también difieren de forma significativa entre chicos y chicas. Las chicas han contestado en mayor medida que nunca lo han hecho, mientras que 
los chicos tienen unos valores significativamente más elevados en que lo hacen por gastar una broma. La literatura previa expone que a menudo, de forma errónea y perjudicial, bromas o apodos realizados de forma continuada son considerados como «buena» convivencia o relación entre amigos (Rawlings, 2019; Shephard et al., 2015). Sin embargo, estas actuaciones para la persona acosada pueden mermar su autoestima y autoconcepto teniendo una influencia negativa en todos los ámbitos de su vida (Norrington, 2020; Romero-Reignier et al., 2016). Estas «bromas» deben de ser atajadas desde el inicio por parte de profesores independientemente el género del que provenga, sin embargo, conocer esta información puede ayudar a focalizar más la atención en este colectivo. A menudo, los propios compañeros también deben de tener la responsabilidad moral de denunciar la situación y no actuar omitiendo la relevancia que puede tener. Para ello, es necesario una concienciación global, la creación de un clima orientado a la tarea y un ambiente de respeto entre los iguales.

Los adultos deben de focalizar la atención para identificar y actuar ante este tipo de situaciones, sin embargo, no es fácil conocerlas en todo momento (Gaffney et al., 2019). Por ello, la educación en valores de los alumnos y alumnas que garantice una actuación adecuada ante la presencia en situaciones de acoso escolar es un aspecto fundamental que hay que trabajar (Prieto et al., 2018). Según los resultados obtenidos, más de la mitad de los chicos ante una situación de acoso escolar trata de cortar la actuación de forma inmediata, un dato significativamente superior que en el caso de las chicas. Sin embargo, las chicas informan a una persona externa (profesorado, familiar, etc.) en mayor medida que los chicos. En cualquier caso, actuar para detener el acoso puede tener efectos positivos en contra del bullying (Ttofi \& Farrington, 2009). Sin embargo, en esta línea, un porcentaje de chicos expresa que no actúa porque cree que no es su problema. Esto tiene lugar de forma significativamente superior que en chicas, en la línea como lo estudiado previamente (Salmivalli, 2001; Salmivalli et al., 2005).

En base a todo lo expuesto según los resultados obtenidos, se pueden exponer diferentes estrategias que pueden ayudar a reducir el bullying y la actuación ante él entre el alumnado. Tradicionalmente esto se ha tratado a través del teatro (Goodwin et al., 2019) o de las artes marciales (Simões et al., 2021) desde el ámbito escolar. Sin embargo, existen estrategias que pueden atacar el problema en líneas generales. Siguiendo algunas de las acciones pedagógicas seguidas por programas de intervención implantados exitosamente cabe destacar una participación activa del alumnado bajo la regla de no aceptar bajo ningún concepto el acoso y hablar la situación. El acoso escolar sería denunciado y castigado (consistente en castigos no punitivos ni castigos físicos). El respeto es el nexo común que debe de existir en una dinámica escolar cálida y educativa. Una propuesta sería abordar esto desde la expresión corporal como terapia de choque. A través del teatro, los alumnos vivenciarán situaciones creadas por el profesorado en las que tengas que actuar como víctimas del acoso escolar, y enfrentar la situación desde un rol que les es ajeno.

\section{Conclusiones}

Las conclusiones que se pueden extraer de este estudio son, en primer lugar, que existen diferencias entre chicos y chicas en algunos aspectos relacionados con la percepción de la problemática del acoso. Por tanto, es un fenómeno donde el análisis de género puede tener sentido para esclarecer algunas incógnitas que ayuden a atajar los problemas.

De forma más concreta, sintetizando los hallazgos, el presente estudio muestra que no existen diferencias entre chicos y chicas en el hecho de sentirse aislado o rechazado en el ámbito escolar, aunque el porcentaje de chicos que nunca se ha sentido rechazado es mayor que el de las chicas. Por otro lado, en cuanto a recibir acoso, la gran mayoría de estudiantes de ambos géneros afirma no haberlo sufrido. Sin embargo, en los que sí lo han sufrido, se aprecia que los chicos reciben acoso mayoritariamente por parte de los chicos, mientras que las chicas lo reciben mayoritariamente por parte de las chicas. En cuanto al acoso que ellos llevan a cabo, no existen diferencias en el hecho de tratar mal a un compañero, aunque hay un porcentaje superior de hombres que dicen meterse más con sus compañeros.

En relación a qué hacen los alumnos cuando se meten mucho con un compañero, sí se han encontrado diferencias, ya que los hombres destacan más en los aspectos de no hacer nada ante esas acciones y meterse más que las chicas para intentar cortar la situación, mientras que las mujeres destacan en informar más a otras personas sobre lo que está sucediendo. En cuanto al lugar donde se producen las situaciones de acoso, no hay diferencias en función el género, pero el porcentaje de chicas que siente que se meten con ellas en clase es superior al de los chicos. Por otro lado, ninguno de los chicos habla con el profesor si ocurren estas acciones de 
acoso, mientras que algunas chicas sí, aunque en porcentajes muy bajos. Atendiendo al hecho de intimidar o maltratar a algún compañero, sí existen diferencias, ya que un mayor número de chicas afirma no meterse con nadie, mientras que un mayor número de chicos justifica su comportamiento argumentando que lo hacen por gastar una broma. Finalmente, podemos asumir que no hay diferencias en función del género ante la pregunta de si tratarían mal a un compañero, aunque el porcentaje de hombres que cree que sí podría llegar a hacerlo es superior. Con esta información se pretende añadir conocimiento sobre el acoso en el ámbito educativo, con la intención de que dicho conocimiento contribuya a la creación de estrategias que permitan atajarlo en mayor medida. A continuación, se muestran las limitaciones del estudio y las futuras líneas de investigación.

\section{Limitaciones y líneas futuras de investiga- ción}

La presente investigación tiene implicaciones para el contexto educativo, ya que el hecho de conocer mejor cómo se produce el acoso escolar y las actuaciones ante este tipo de situaciones puede aportar información útil. En este sentido, conocer dicha información puede permitir a los docentes tratar de atajar la aparición del problema de mejor manera, pudiendo plantear estrategias que atiendan a las peculiaridades que van mostrando las investigaciones de la temática. En cuanto a las limitaciones, lógicamente el hecho de analizar un centro educativo hace que los resultados se ajusten a una realidad muy concreta, por lo que en investigaciones futuras sería interesante realizar un análisis de esta temática más amplio que abarque distintos centros. Por otro lado, también sería interesante tratar de agrupar en una medición, centros de distinta titularidad y localización, atendiendo a entornos urbanos y rurales, de cara a tratar de identificar posibles variaciones en función de dichas características, lo que podría ayudar a definir de forma más concreta las estrategias para la prevención del acoso.

\section{Referencias}

Alonso, N. J. M. L., Gómez, R. L., Córcoles, M. Á.A., Sáiz, J. C. B., \& García, G. G. J. (2017). Acoso Escolar: Desde la sensibilización social a una propuesta de intervención. Reflexiones desde la legislación española. Letras Jurídicas, 4(4), 1-16.

Bailey, R. (2006). Physical education and sport in schools: A review of benefits and outcomes. Journal of School Health, 76(8), 397-
401. https://doi.org/10.1111/j.1746-1561.2006.00132.x Ballesteros, R. (2018). Mapa del acoso escolar: España supera por primera vez las mil víctimas en un año. El Confidencial. Recuperado en 13 de junio de 2019, de: https:// www.elconfidencial.com/espana/2018-10-18/mapa-acoso-escolar-espana-mil-victimas-ano_1631192/

Bascón-Seda,A. \& Ramírez-Macías, G. (2020).Víctimas de bullying: aportaciones para identificar casos de acoso en Educación Física dentro de laEducación Secundaria Obligatoria. Cultura, Ciencia y Deporte, 15(43), 109-119.

Bejerot, S., Plenty, S., Humble,A., \& Humble, M. B. (2013). Poor motor skills: A risk marker for bully victimization. Aggressive Behavior, 39(6), 453-461. https://doi.org/10.1002/ ab.21489

Brandão Neto,W., Silva, C. O. da,Amorim, R. R.T.do,Aquino, J.M. de,Almeida Filho,A.J.de, Gomes, B. da M. R., \& Monteiro, E. M. L. M. (2020). Formation of protagonist adolescents to prevent bullying in school contexts. Revista Brasileira de Enfermagem, 73(1), 1-8. https://doi.org/10.1590/00347167-2019-0418

Castañeda-Vázquez, C., Moreno-Arrebola, R., González-Valero, G.,Viciana-Garófano,V., \& Zurita-Ortega, F. (2020). posibles relaciones entre el bullying y la actividad física: una revisión sistemática. Journal of Sport \& Health Research, 12(1).

Cerezo-Ramírez, F. (2012). Bullying a través de las TIC. Boletín Cientifico Sapiens Research, 2(2), 24-29.

Fernández, I., \& Ortega, R. (1999). Cuestionario sobre abusos entre compañeros. Prevención de La Violencia y Resolución de Conflictos. El Clima Escolar Como Factor de Calidad, 210 219.

Fernández, M. J. M., \& Vivar, D. M. (2017). La escuela inclusiva ante el acoso escolar Estrategias de prevención para el profesorado. Revista de Educación Inclusiva, 3(3), 123-133.

Fialho, N., \& Bakshi, A. J. (2016). Understanding school bullying: its nature and prevention strategies. British Journal of Guidance \& Counselling, 44(2), 246-248, DOI: 10.1080/ 03069885.2015.1070635

Gaffney, H., Farrington, D. P., \& Ttofi, M. M. (2019). Examining the effectiveness of school-bullying intervention programs globally: a meta-analysis. International Journal of Bullying Prevention, 1(1), 14 -31. https://doi.org/10.1007/s42380019-0007-4

Garandeau, C. F., Laninga-Wijnen, L., \& Salmivalli, C. (2021). Effects of the Kiva anti-bullying program on affective and cognitive empathy in children and adolescents. Journal of Clinical Child \&Adolescent Psychology, 1-15.https: / /doi.org/10.1080/ 15374416.2020.1846541

Gómez, J. M. R. (2009). Acoso escolar-Medidas de prevención y actuación. Educação, 32(1), 51-58.

Goodwin, J., Bradley, S. K., Donohoe, P., Queen, K., O’Shea, M., \& Horgan,A. (2019). Bullying in schools: an evaluation of the use of drama in bullying prevention. Journal of Creativity in Mental Health, 14(3), 329-342. https://doi.org/10.1080/ 15401383.2019.1623147 
Green,V.A.,Woods, L., Wegerhoff, D., Harcourt, S., \& Tannahill, S. (2020). An evaluation of the KiVa anti-bullying program in New Zealand. International Journal of Bullying Prevention, 2(3), 225-237. https://doi.org/10.1007/s42380-019-000346

Izquierdo, A. J. C. (2017). Perfil del acoso escolar en institutos de enseñanza secundaria de zonas rurales y urbanas en Andalucía. Revista de Derecho, Empresa y Sociedad (REDS), 10, 159-171.

Jiménez-Barbero, J. A., Jiménez-Loaisa, A., González-Cutre, D., Beltrán-Carrillo,V.J., Llor-Zaragoza,L., \& Ruiz-Hernández, J. A. (2020). Physical education and school bullying: a systematic review. Physical Education and Sport Pedagogy, 25(1), 79-100. https: / /doi.org/10.1080/17408989.2019.1688775

Knauf, R.-K., Eschenbeck, H., \& Hock, M. (2018). Bystanders of bullying: Social-cognitive and affective reactions to school bullying and cyberbullying. Cyberpsychology:Journal of Psychosocial Research on Cyberspace, 12(4), 1-17. https://doi.org/10.5817/ CP2018-4-3

Larrain, E., \& Garaigordobil, M. (2020). El bullying en el País Vasco: prevalencia y diferencias en función del sexo y la orientación sexual. Clínica y Salud, 31(3), 147-153. https: / / dx.doi.org/10.5093/clysa2020a19

Limber, S. P., Olweus, D., Wang,W., Masiello, M., \& Breivik, K. (2018). Evaluation of the Olweus Bullying Prevention Program: A large scale study of US students in grades 3-11. Journal of School Psychology, 69, 56-72. https://doi.org/ 10.1016/j.jsp.2018.04.004

Marracho,P., de Castro, E.M.R.T., da Graça Nery, M.V., \& Pereira, A. M. A. (2021). Comportamentos de bullying em jovens praticantes de desporto (Bullying behaviours in young athletes). Retos, 42, 861-871

Martínez-Baena,A., \& Faus-Boscá, J. (2018). Acoso escolar y Educación Física: una revisión sistemática. Retos, 34, 338-345.

Martínez-Baena,A., \& Faus-Boscá, J. (2018). Acoso escolar y Educación Física: una revisión sistemática. Retos, 34, 338-345.

Menéndez Santurio, J. I., \& Fernández Río, F. J. (2018). Actitudes hacia la violencia y papel importante del alumnado en el aula de educación física. Revista complutense de educación, 29(4), 1293 1208.

Navarrete-Galiano, R. (2009). Los medios de comunicación y la concienciación social en España frente al acoso escolar. Estudios Sobre El Mensaje Periodístico, 15, 335-345. http:// hdl.handle.net/11441/29258

Norrington, J. (2020).Adolescent peer victimization, self-concept, and psychological distress in emerging adulthood. Youth \& Society, 53(2), 273-295. https://doi.org/10.1177/ 0044118 X20910938

Olweus, D. (1998). Conductas de acoso y amenaza entre escolares. Ediciones Morata, Madrid, España.

Prieto,A.,Hernández,J. J., \& Enríquez,E. G. (2018). La formación de valores desde la clase de educación física. PODIUM: Revista de Ciencia y Tecnología En La Cultura Física, 13(1), 7487.

Rawlings, V. (2019). 'It's not bullying' ,It's just a joke':Teacher and student discursive manoeuvres around gendered violence. British Educational Research Journal, 45(4), 698-716. https: / / doi.org/10.1002/berj.3521

Romero-Reignier, V., Prado, V., \& Soto-Rubio, A. (2016). La influencia del bullying en la autoestima de los adolescentes. Calidad deVida y Salud, 9(1), 2-12.

Salmivalli, C. (2001). Peer-led intervention campaign against school bullying: who considered it useful, who benefited? Educational Research, 43(3), 263-278. https://doi.org/10.1080/ 00131880110081035

Salmivalli, C., Kaukiainen, A., \& Voeten, M. (2005). Anti-bullying intervention: Implementation and outcome. British Journal of Educational Psychology, 75(3), 465-487. https://doi.org/ 10.1348/000709905X26011

Shephard, B., Ordóñez, M., \& Oleas, C. M. (2015). Estudio descriptivo: programa de prevención y disminución del acoso escolar-»bullying». Fase diagnóstica: prevalencia. Revista Médica HJCA, 7(2), 155-161. http://dx.doi:10.14410/ 2015.7.2.ao.30.

Shiakou, M., Stella,H., \& Georgia,Y. (2019). Understanding bullying through the eyes of children. International Journal on School Climate and Violence Prevention, 3(1), 157-170.

Simões, H., Santos, P. M., Pereira, B., \& Figueiredo, A. (2021). As Artes Marciais e os Desportos de Combate e o Bullying: uma revisão sistemática Martial Arts and Combat Sports and the Bullying: a systematic review. (Las artes marciales y deportes de combate y el acoso escolar: una revisión sistemática). Retos, 39, 835-843.

Toscano, J.A., Álvarez, E., Rambal, L., Vargas, L., Ávila-Toscano, J. H., Fontalvo, E. Á., Rambal-Rivaldo, L. I., \& Vargas-Delgado, L. (2020). Importancia de los estilos de socialización parental en los roles del acoso entre pares. Interdisciplinaria. Revista de Psicología y Ciencias Afines, 38(1), 203-216. https://doi.org/ 10.16888/interd.2021.38.1.13

Ttofi, M., \& Farrington, D. (2009). What works in preventing bullying: Effective elements of anti-bullying programmes. Journal of Aggression, Conflict and Peace Research, 1(1), 1324. https://doi.org/10.1108/17596599200900003

Vega-Osés, A., \& Peñalva-Vélez, A. (2018). Los protocolos de actuación ante el acoso escolar y el ciberacoso en España: un estudio por comunidades autónomas. International Journal of New Education, 1(1), 1-26. DOI: 10.24310/ IJNE1.1.2018.4971

Vélez, S. C., \& Fernández, J.A. Á. (2018). El maltrato escolar en centros de educación primaria en la provincia de Huelva (España). Psicodebate. Psicología, Cultura y Sociedad, 18(1), 51-71.

Voors, W. (2005). Bullying, el acoso escolar:el libro que todos los padres deben conocer (Vol. 65), Oniro.

Wachs, S., Bilz,L., Niproschke, S., \& Schubarth,W. (2019). Bullying intervention in schools: A multilevel analysis of teachers' success in handling bullying from the students' perspective. The Journal of Early Adolescence, 39(5), 642-668. https://doi.org/ 10.1177/0272431618780423

Zambuto,V., Palladino, B.E., Nocentini,A., \& Menesini,E.(2020). Voluntary vs nominated peer educators: A randomized trial within the NoTrap! Anti-Bullying Program. Prevention Science, 21(5), 639-649. 